\title{
Theoretical Justification of Plant Stems Vibro-Cutting in Dense Environment
}

\author{
A.P. Tarverdyan, A.Kh. Khachatryan, A.V. Altunyan \\ Armenian National Agrarian University \\ arshaluystar@gmail.com, aghavard59@mail.ru, artur_altunyan@mail.ru
}

\section{A R T I C L E I N F O}

Keywords:

water environment, vibro-cutting, resistance forces, damped oscillations, resistance reduction

\begin{abstract}
A B S T RA C T
The article considers the issue related to the disclosure of the reasons for the abrupt reduction of resistance force factors in case of plants stems vibro-cutting in dense medium through theory-based investigations.

The computation scheme of the liquid motion in the vicinity of vibro-blade has been recommended, which enabled to derive differential equation of the motion resulted under the impact of interaction forces between the vibro-blade and environment.

It has been proved, that the liquid in the vibro-blade vicinity is subjected to rapid damped oscillation, due to which the environmental resistance forces and the energy consumption rates are reduced in about 20 times against the same indices recorded in case of vibrationless cutting.
\end{abstract}

\section{Introduction}

In the previous three scientific reports (Tarverdyan ${ }^{1)}$, et al., 2020, Tarverdyan ${ }^{2}$, et al., 2020, Tarverdyan ${ }^{3)}$, et al., 2020), the relevance and urgency of conducting comprehensive investigations on the plants stem cutting in the dense medium (water, soil) is thoroughly justified.

Particularly, the cleaning of reservoirs and canals from the cane-like and other water plants is an important issue. The practice of applying the current segmented-finger cutting apparatus has indicated that they aren't so much efficient for the use in aquatic environment.
In this regard rotary cutting apparatus are more preferable, anyhow, they haven't provided the desired results either (Tarverdyan, 1996, Tarverdyan, 2014, CSRIITE, 1978). The blade speed of the currently applied rotary cutting apparatus makes 30-50 m/s (Tarverdyan, 1996, Blinov, 1973); the mentioned speed generates such high resistance forces, which result in rapid reduction of rotation numbers in the rotors. In the result of investigations, it has been found out that the increase of the rotation numbers only in two times requires 5 times more power consumption (Tarverdyan ${ }^{1)}$, et al., 2020, Tarverdyan ${ }^{2}$, et al., 2020, Tarverdyan, 2014). 
The attempts aimed at upgrading of cutting apparatus have been doomed to failure (CSRIITE, 1978); this means that the design of a completely new apparatus is the only way to handle the raised problem. Upon the results of longterm experiments on the plants stem (both thin- and stiff thick-stalked) cutting, it has been proved that it is possible to implement cutting in the dense environment with the minimum energy consumption using a vibro-blade which receives vibrational motion with low amplitude $(2 \div 8 \mathrm{~mm})$ and relatively higher frequency $\left(30 \div 100 \mathrm{~s}^{-1}\right)$, while the rotation numbers transmitted to the rotor is relatively low making up to $1.0 \mathrm{~s}^{-1}$ (Tarverdyan, 1996, Tarverdyan, 2014, Altunyan, 2009).

The small rotation numbers in the rotor and, hence, the small circumferential velocities don't generate additional resistance forces in the dense environment, while the stem cutting resistance forces sharply drop down in the result of vibrational movements of the blades (Tarverdyan ${ }^{1)}$, et al., 2020, Tarverdyan, 1996, Altunyan, 2009).

\section{Materials and methods}

Throughout the studies of field-related scientific literature no research work devoted to the theoretical research on the plant stems vibratory cutting in the dense medium has been ever found. To this end we have set a task to comprehensively study the vibro-cutting mechanisms in the dense environment and to try to reveal the causes for rapid reduction of vibro-blade resistance forces in the dense environment.

First, the plants stem vibrationless cutting in the water medium, and then cutting with vibratory blade movement in the same conditions have been considered. The solution to the first mentioned problem is thoroughly introduced in the first article of the current series (Tarverdyan ${ }^{1}$, et al., 2020), i.e., the cutting process of the cane stem in water environment with the blade of the rotary cutting apparatus without blade's vibration has been examined. A specific computational pattern has been selected and by using the well-known principles and laws of hydrodynamics (MilneThomson, 1964, Prandtl, 2000), all environmental resistance force factors affecting the blade have been determined. In the second stage of the problem solution, the vibratory cutting of the cane stems in water medium has been investigated (Tarverdyan²), et al., 2020).

Upon the experiments it has been asserted that it is relevant to implement the plants stem cutting in the dense medium along the mutually perpendicular directions of the blade cutting edge in conditions of balanced oscillations (Tarverdyan, 2014). So, to identify the specifics of vibrocutting, the blade vibration mode should be chosen through the Elliptic law (Tarverdyan, 1996, Tarverdyan, 2014, Bolotin, 1978, Levendel, 1981). To disclose the effect of vibratory movement on the resistance forces of the water medium, a computation scheme has been selected (Tarverdyan ${ }^{2}$, at al., 2020) and an assumption has been made according to which the water mass within the range of elementary prism, shifts the movement direction during a single oscillation phase (the vibration frequency) resulted from the vibratory movement, and hence, the epure of the fluid motion velocities in the perpendicular directions of the blade sheet will look like the diagram introduced in Figure 1 a.

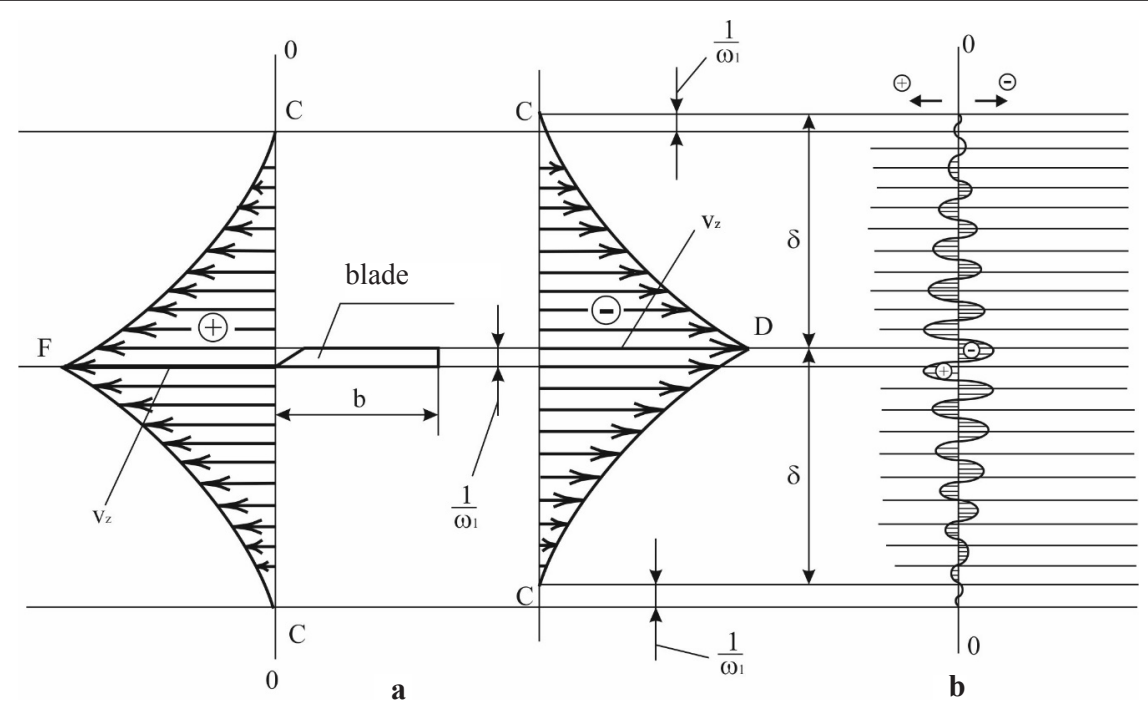

Figure 1. The epure of the fluid motion velocities in the vicinity of the vibro-blade moving in the liquid (composed by the authors). 
The epure overview of the fluid particle velocities in the vicinity of vibro-blade is introduced in Figure $1 \mathrm{~b}$.

It is easily noticed that the total area of the summary epure can be practically assumed as zero, moreover, the higher vibration frequency is $\left(\omega_{1},\right)$, the more reliable the abovementioned assumption becomes. So, if the volume of the vibration-driven moving liquid is practically equal to 0 (the area of the elementary prism base: $A \rightarrow 0$ ), then the moving liquid mass $-d m=0$ - and all the force factors ( $T_{x}$ and $P_{i n}$ ), which are related to the mass flow of the moving liquid and generate resistance forces of the environment, are practically turned to 0 (Tarverdyan ${ }^{2)}$, et al., 2020).

In case of vibration, from the resistance force factors in the blade movement, only resistance momentum is available, the value of which depends on forces (Tarverdyan ${ }^{1)}$, et al., 2020), which is reduced in 10-35 times (Tarverdyan ${ }^{2}$, et al., 2020).

The recommended model and computation scheme for the problem solution have enabled to disclose the reasons for the abrupt decrease in the resistance forces of the water medium.

It is noteworthy that the received results are based on the abovementioned assumption and precise solution of the problem is of high priority, first, from the prospect of proving the assumption and then from that of revealing the specifics of vibro-cutting. Based on the afore stated and on the view of epure designed for the vertical water motion against the upper and lower vibro-blade sheets, it becomes logical to find the precise solution to the problem within the scope of the damped oscillation theory (Biderman, 1980).
As in previous cases (Tarverdyan ${ }^{1)}$, et al., 2020, Tarverdyan ${ }^{2}$, et al., 2020), here again, let's choose a design diagram which articulates the real state of the interactive forces in fluid motion, moving mass, vibro-blade and water environment and their regularities more accurately. When choosing the computation pattern (Figure 2), the well-known hydrodynamic provisions (Milne-Thomson, 1964, Prandtl, 2000) and the diagrams discussed in the previous works (Tarverdyan ${ }^{1)}$, et al., 2020, Tarverdyan ${ }^{2}$, et al., 2020) have been taken into account, the theoretical findings of which have been proved through scientific experiments with sufficient precision.

Since the rotational movement (shifting) of the blade hardly generates environmental resistance forces, which has been reasonably stated above, only vibration movement has been considered when designing the computation scheme.

The most significant difference against the previous schemes is that the fluid movement is performed only along the vibro-blade latitude $\mathrm{b}$ and longitude $\ell$, since, as it has been already mentioned above, $\omega_{0}=0$. The letter designations of the values are the same as in the previous schemes (Tarverdyan ${ }^{1)}$, et al., 2020, Tarverdyan'), et al., 2020).

So, $\omega_{0}$ is the rotor's rotation frequency, $a_{x}$ is the oscillation amplitude along the length of the blade cutting edge, $a_{z}$ is the oscillation amplitude perpendicular to the blade cutting edge, $V_{x}$ is the vibration velocity towards the $x$ axis $\left(V_{x}=a_{x} \omega_{1} \cos \omega_{1} t\right), V_{z}$ is the vibration velocity towards the $\mathrm{z}$ axis $\left(V_{z}=a_{z} \omega_{1} \sin \omega_{1} t\right), \omega_{1}$ is the vibration frequency in mutually perpendicular directions $\left(\omega_{x}=\omega_{z}=\omega_{1}\right)$.

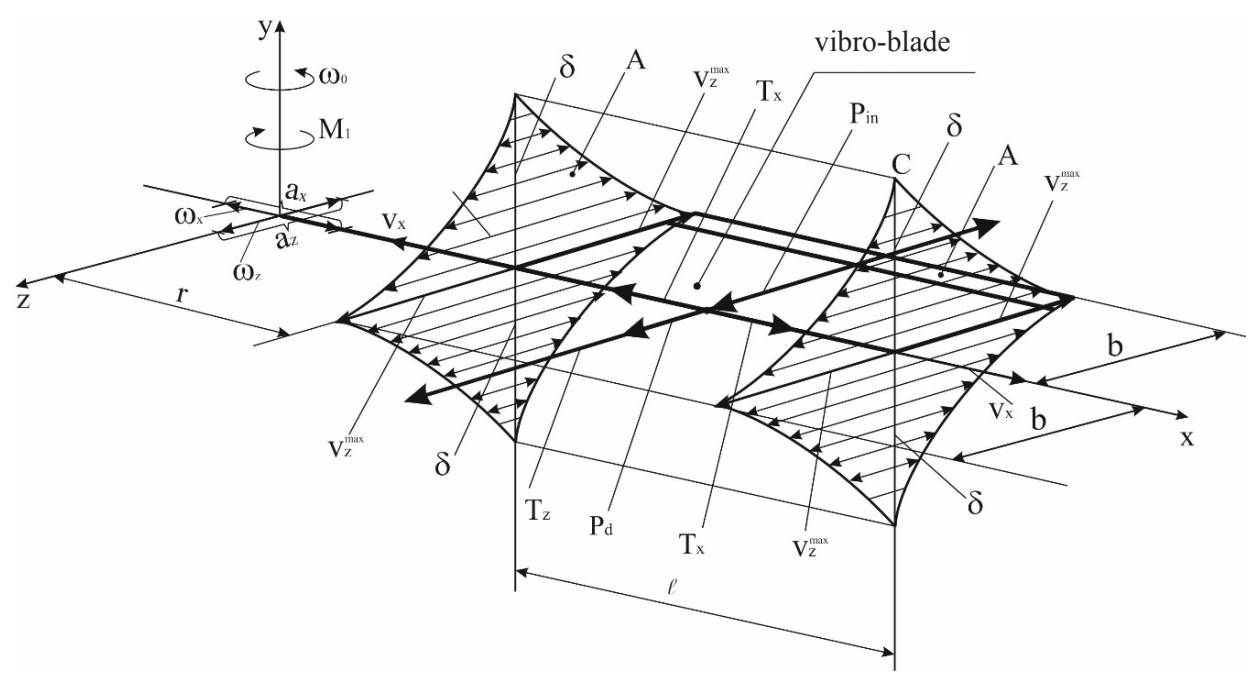

Figure 2. The computation scheme for the determination of the resistance forces in the vibro-blade movement of the cutting apparatus in water environment (composed by the authors). 
In the considered case the liquid mass in movement will be:

$$
M=A \cdot \ell \cdot \rho
$$

where $A$ is the area of the prism built with four parabolic triangles $A=4 \cdot \frac{1}{3} b \cdot \delta, \rho$ is the liquid density.

By inserting we'll have:

$$
M=\frac{4}{3} b \cdot \delta \cdot \ell \cdot \rho .
$$

The interactive force factors of the moving fluid mass and vibro-blade are as follows (Tarverdyan ${ }^{1)}$, et al., 2020, Tarverdyan ${ }^{2)}$, et al., 2020):

- Tangential resistance force towards the latitudinal direction of the vibro-blade sheet: $T_{z}$

$$
T_{z}=6 b \ell \sqrt{\mu \rho \omega_{1}} \frac{d z}{d t} .
$$

- Tangential resistance force towards the longitudinal direction of the vibro-blade sheet: $T_{x}$

$$
T_{x}=\frac{4}{3} \rho \omega_{1} \sqrt{\frac{v b \ell}{\omega_{1}}} \cdot \frac{2}{5} \ell \cdot \frac{d x}{d \ell} .
$$

- Hydrodynamic resistance force, which is directed to the blade width vertical to the cutting edge: $P_{d}$.

$$
P_{d}=c \cdot \lambda \cdot \rho \cdot \ell^{2} \cdot z
$$

- Inertia forces towards the $Z$ and $X$ axes: $P_{i n(z)}$ and $P_{i n(x) \text {. }}$

$$
P_{i n(z)}= \pm M \cdot \frac{d^{2} z}{d t^{2}}, \quad P_{i n(x)}= \pm M \cdot \frac{d^{2} x}{d t^{2}} .
$$

The letter designations and their numerical values in the above mentioned expressions are as follows for our problem: $\rho$ is the environmental density $\left(1000 \mathrm{~kg} / \mathrm{s}^{3}\right.$, this and other values refer to water medium), $\mu$ is the viscosity coefficient $(0.1 \mathrm{~kg} / \mathrm{m} \cdot \mathrm{s}), v$ is the kinematic viscosity coefficient $\left(1 \cdot 10^{-6} \mathrm{~m}^{2} / \mathrm{s}\right), c$ is the constant coefficient, it depend on the blade shape and sizes (in our case $c=1.45$ (Prandtl, 2000), $b$ is the width of the blade sheet $(0.03 \mathrm{~m})$, $\ell$ is the length of the blade sheet (cutting edge) $(0.3 \mathrm{~m})$, $\lambda$ is the thickness of the blade sheet $(0.001 \mathrm{~m})$.

\section{Results and discussions}

From the prospect of discussed problem the force factors, which are directed towards the $Z$ axis and generate resistance moment against the rotor's shaft of applied cutting apparatus, whereupon the value of applied power is determined, are of primary interest. For the $M$ mass of the liquid the Newton second law will look as follows:

$M \frac{d^{2} z}{d t^{2}}=-M \frac{d^{2} z}{d t^{2}}-6 b \ell \sqrt{\mu p \omega_{1}} \cdot \frac{d z}{d t}-c \cdot \lambda \cdot \omega_{1}^{2} \cdot p \cdot \ell^{2} \cdot z,(1)$

or by placing the M value we'll have:

$$
\frac{8 p \delta b \ell}{3} \cdot \frac{d^{2} z}{d t^{2}}+6 b \ell \sqrt{\mu p \omega_{1}} \frac{d z}{d t}+c \cdot \lambda \cdot \omega_{1}^{2} \cdot p \cdot \ell^{2} \cdot z=0 .
$$

Here is the differential equation of the moving liquid mass, which enables to describe the damping oscillations in case of some parametric values.

It is worth mentioning that the expression of $6 b \ell \sqrt{\mu \rho \omega_{1}}$ is the damping coefficient $\left[\frac{\mathrm{kg}}{\mathrm{s}}\right]$, and $c \cdot \lambda \cdot \omega_{1}^{2} \cdot \rho \cdot \ell^{2}$ is the coefficient of elastic resistance $\left[\frac{\mathrm{kg}}{\mathrm{s}^{2}}\right]$.

The expression (2) will look as follows:

$$
\frac{d^{2} z}{d t^{2}}+\frac{9 \sqrt{\mu \rho \omega_{1}}}{4 \rho \delta} \cdot \frac{d z}{d t}+\frac{3 c \cdot \lambda \cdot \omega_{1}^{2} \cdot \ell}{8 \delta b} \cdot z=0 .
$$

In this expression $\mu, \rho, c, \lambda, \ell, b$ are constant values, $\omega_{1}$ and $\delta$ are also constant values in each considered case, anyhow, since one of the research objectives is the study of convergence just related to $\omega_{1}$, then by assigning it with arbitrary values, we'll get the damping character and parameters within the identified range of the vibro-blade action $\left(30 \div 100 \mathrm{~s}^{-1}\right)$.

The selected value for $\omega_{1}$ determines the value of $\delta$ (the height of liquid strata in motion) (Tarverdyan ${ }^{1)}$, et al., 2020, Tarverdyan ${ }^{2}$, et al., 2020):

$$
\delta=\sqrt{\frac{\mu b}{V_{z}^{\max }}}
$$

where $V_{z}^{\max }$ is the maximum value of vibration speed towards the latitudinal blade direction: $V_{z}^{\max }=a_{z} \cdot \omega_{1}$.

By inserting the numerical values of the constants in (3), we'll have:

$$
\frac{d^{2} z}{d t^{2}}+7.5+10^{-3} \frac{\sqrt{\omega_{1}}}{\delta} \cdot \frac{d z}{d t}+1.813 \cdot 10^{-3} \cdot \frac{\omega_{1}^{2}}{\delta} \cdot z=0 .
$$

Let's assign:

$$
m=7.5 \cdot 10^{-3} \frac{\sqrt{\omega_{1}}}{\delta} \text { and } n=1.813 \cdot 10^{-3} \frac{\omega_{1}^{2}}{\delta} .
$$

In each considered case, when $\omega_{1}$ and consequently $\delta$ have certain values, $m$ and $n$ are constant and positive. 
Let's find the solution of the differential equation (4) in the following form: $z(t)=e^{k t}$.

The descriptive equation will be:

$$
k^{2}+m k+n=0
$$

For the oscillations to be convergent, the following term should be satisfied: $m^{2}-4 n<0$.

In that case the equation (6) will have complex roots:

$$
\begin{gathered}
k_{1}=-\alpha+i \beta \text { and } k_{2}=-\alpha-i \beta, \\
\frac{d^{2} z}{d t^{2}}+m \frac{d z}{d t}+n \cdot z=0 .
\end{gathered}
$$

The general solution of the equation will look as follows:

$$
z(t)=e^{-\alpha t}\left(C_{1} \cos \beta t+C_{2} \sin \beta t\right),
$$

where $C_{1}$ and $C_{2}$ constants are determined upon the following initial conditions: $z(0)=\alpha_{1}$ and $z^{\prime}(0)=\beta_{1}$.

It is evident that for any $C_{1}$ and $C_{2}$ cases such $\mathrm{A}$ and $\varphi$ values can be chosen so as to have the following:

$$
\begin{aligned}
& C_{1}=A \sin \varphi, \quad C_{2}=A \cos \varphi, \\
& A=\sqrt{C_{1}^{2}+C_{2}^{2}}, \quad \varphi=\operatorname{arctg} \frac{C_{1}}{C_{2}} .
\end{aligned}
$$

The (7) expression can be presented in the following way: $z(t)=A \cdot e^{-\alpha t}(\sin \varphi \cos \beta t+\cos \varphi \sin \beta t)=A e^{-\alpha t} \cdot \sin (\beta t+\varphi)$.

The (9) expression is the oscillation equation, the initial amplitude of which is $A$, and $\varphi$ is the initial phase, which are determined through the (8) expression.

Let's determine the $C_{1}$ and $C_{2}$ constants.

From the term of $t=0$ it follows that $C_{1}=a_{1}$.

$$
\begin{gathered}
z^{\prime}(t)=-\alpha \cdot e^{-\alpha t}\left(C_{1} \cos \beta t+C_{2} \sin \beta t\right)+ \\
+e^{-\alpha t} \cdot\left(-\beta C_{1} \sin \beta t+\beta C_{2} \cos \beta t\right) . \\
z^{\prime}(0)=-\alpha C_{1}+\beta C_{2}=\beta_{1},
\end{gathered}
$$

wherefrom $\quad C_{2}=\frac{\beta_{1}+a \cdot a_{1}}{\beta}$.

By placing in the (8) expression, we'll have:

$$
A=\sqrt{a_{1}^{2}+\left(\frac{\beta_{1}+a \cdot a_{1}}{\beta}\right)^{2}}, \quad \varphi=\operatorname{arctg} \frac{a_{1} \cdot \beta}{\beta_{1}+a \cdot a_{1}} .
$$

From the practical viewpoint the state of damped oscillation depending on fluctuation $\omega_{1}$ frequency and the blade geometric parameters is of great significance.
Let's determine the $C_{1}$ and $C_{2}$ constants based on the initial terms relevant to our problem. At the beginning of the vibroblade movement we have $(t=0), z(0)=0$, from which it follows, that $C_{1}=\alpha_{1}=0$. The maximum oscillation velocity is in the point of $z=0$ therefore $z^{\prime}(0)=V_{z}^{\max } \equiv\left(\beta_{1}\right)=c_{2} \cdot \beta$ wherefrom $C_{2}=\frac{V_{z}^{\max }}{\beta}$.

In case of initial parameters of our problem the equation of damped oscillation will take the following form:

$z(t)=C_{2} e^{-\alpha t} \cdot \sin \beta t$ or $z(t)=\frac{a_{z} \cdot \omega_{1}}{\beta} \cdot e^{-a t} \cdot \sin \beta t$.

For each considered case it is necessary to determine $\alpha$ and $\beta$.

Let's consider the following options:

\section{1. $\omega_{1}=30 \mathrm{~s}^{-1}:$ in this case}

$$
\begin{gathered}
\delta_{i}=5.8 \cdot 10^{-3} \mathrm{~m}, V_{z}^{\max }=0.09 \mathrm{~m} / \mathrm{s}, \\
m=7.5 \cdot 10^{-3} \frac{\sqrt{30}}{5.8 \cdot 10^{-3}}=7.08 \mathrm{~s}^{-1}, \\
n=1.813 \cdot 10^{-3} \cdot \frac{900}{5.8 \cdot 10^{-3}}=281 \mathrm{~s}^{-2} .
\end{gathered}
$$

The descriptive equation will be: $k_{2}+7.08 k+281=0$.

$$
\begin{gathered}
k_{1,2}=-3.54 \pm 16.385 i, \alpha=-3.54, \beta=16.385 . \\
A=\frac{0.09}{16.385}=0.00549 \quad m=0.549 \mathrm{~cm} .
\end{gathered}
$$

The diagram of the damped oscillation function $z(t)=0.549 e^{-3,54 t} \cdot \sin (16.385 t)$ is introduced in Figure 3.

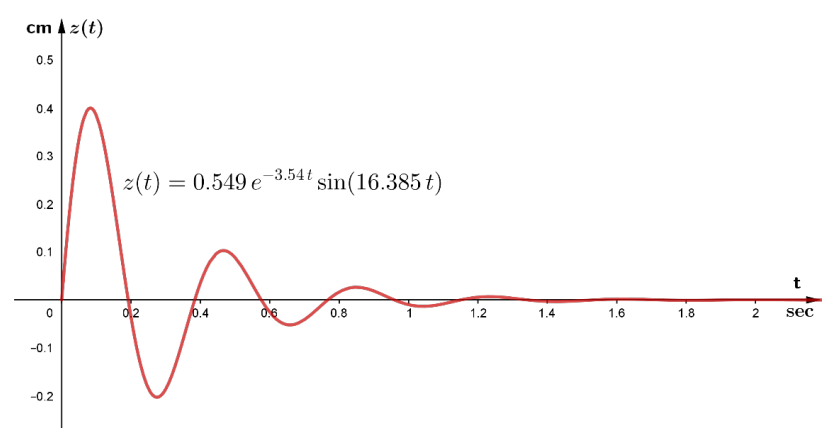

Figure 3. Diagram of $z(t)=0.549 e^{-3,54 t} \cdot \sin (16.385 t)$ function. (composed by the authors). 


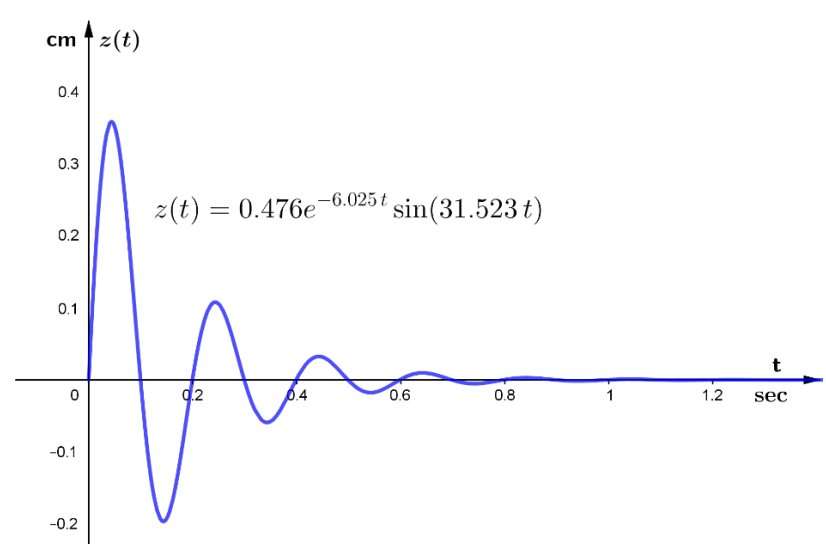

Figure 4. Diagram of $z(t)=0.476 e^{-6.025 t} \cdot \sin (31.523 t)$ function (composed by the authors).

\section{2. $\omega_{1}=50 \mathrm{~s}^{-1}$ : in this case}

$$
\begin{gathered}
\delta_{i}=4.4 \cdot 10^{-3} \mathrm{~m}, V_{z}^{\max }=0.15 \mathrm{~m} / \mathrm{s}, \\
m=12.05 \mathrm{~s}^{-1}, \quad n=1030 \mathrm{~s}^{-2}, \quad k_{1.2}=-6.025 \pm 3.523 i, \\
\alpha=-6.025, \beta=31.523, A=0.476 \mathrm{~cm} .
\end{gathered}
$$

The diagram of $z(t)=0.476 e^{-6.025 t} \cdot \sin (31.523 t)$ function is introduced in Figure 4.

\section{3. $\omega_{1}=100 \mathrm{~s}^{-1}:$ in this case}

$$
\begin{gathered}
\delta_{i}=0.0032 \mathrm{~m}, V_{z}^{\max }=0.3 \mathrm{~m} / \mathrm{s} \\
m=23.43 \mathrm{~s}^{-1}, \quad n=5664 \mathrm{~s}^{-2}, \\
k_{1,2}=-11.71 \pm 74.342 i, \quad \alpha=-11.715, \\
\beta=74.342, A=0.404 \mathrm{~cm} .
\end{gathered}
$$

The diagram of $z(t)=0.404 e^{-11.715 t} \cdot \sin (74.342 t)$ function is introduced in Figure 5.

4. Let's also consider the effect of vibro-blade geometrical parameters on the convergence of oscillations. For the second variant $\left(\omega_{1}=50 \mathrm{~s}^{-1}\right)$ let's assume that $b=0.05 \mathrm{~m}$ and $\lambda=0.002 \mathrm{~m}$. We'll have:

$$
\begin{gathered}
m=12.05 \mathrm{~s}^{-1}, \quad n=1235 \mathrm{~s}^{-2}, \\
k_{1,2}=-6.025 \pm 34.622 i, \alpha=-6.025, \\
\beta=34.622, A=0.433 \mathrm{~cm} .
\end{gathered}
$$

The diagram of $z(t)=0.433 e^{-6.025 t} \cdot \sin (34.622 t)$ function is introduced in Figure 6.

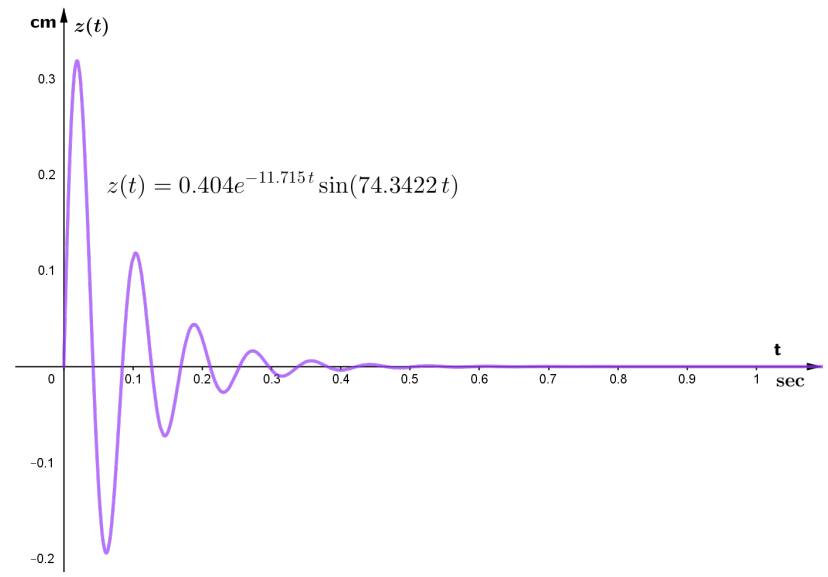

Figure 5. Diagram of $z(t)=0.404 e^{-11.715 t} \cdot \sin (74.342 t)$ function (composed by the authors).

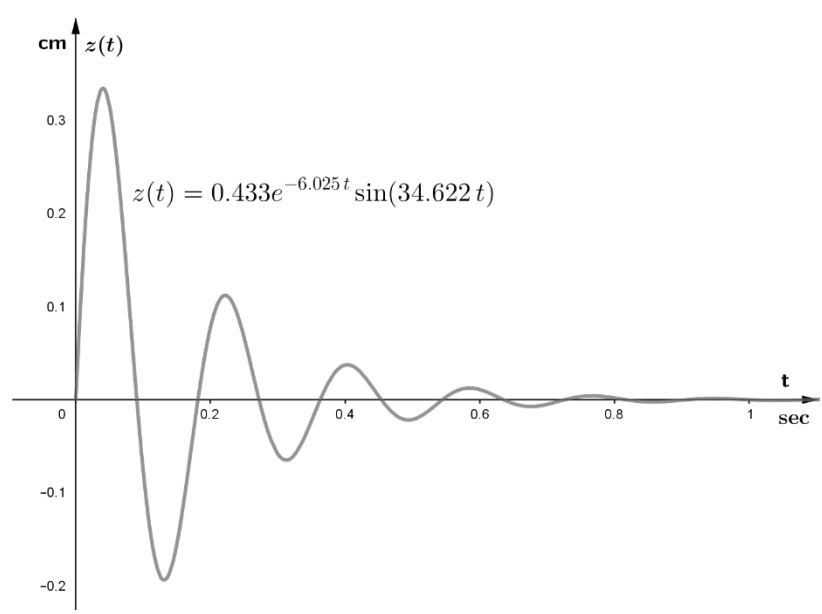

Figure 6. Diagram of $z(t)=0.433 e^{-6.025 t} \cdot \sin (34.622 t)$ function (composed by the authors).

Thus, based on the results of software solution of the equations and the oscillogram analyses, it can be stated that the water mass around the vibro-blade vicinity is subjected to fast damping oscillation in the water medium, which entails to the abrupt decrease in the environmental resistance forces.

\section{Conclusion}

Upon the precise problem solution the hypothesis that the fluid of the vibro-blade vicinity is subjected to the damped oscillation has been proved and justified; so the blade resistance forces in the dense medium are sharply reduced $(10 \div 30$ times $)$. 
The damping time of the oscillations $(t)$ is inversely proportional to the vibro-blade oscillation frequency $\left(\omega_{1}\right)$. For example, if $\omega_{1}=30 \mathrm{~s}^{-1}$, then $t=1.5 \mathrm{~s}$ (Figure 3), if $\omega_{1}=50 \mathrm{~s}^{-1}$, then $t=0.9 \mathrm{~s}$, (Figure 4), if $\omega_{1}=100 \mathrm{~s}^{-1}$, then $t=0.45 \mathrm{~s}$ (Figure 5), which is quite logical and affirms the compatibility of background assumptions for the problem solution and the computation scheme with the real character of vibro-blade and water medium interactions.

The geometrical dimensions of the vibro-blade have no significant effect on the specifics and parameters of oscillation damping.

\section{References}

1. Altunyan, A.V. (2009). Development of Technologies and a Working Part for Cutting Stems in Dense Environment. PhD, Yerevan (in Armenian).

2. Biderman, V.A. (1980). The Theory of Mechanical Oscillations. Publish. "Vishaya Shkola", M., - 405 p. (in Russian).

3. Blinov, Yu. (1973). Development of Designs for Rotary Mowers (Foreign Practice) // Engineering in Agriculture, Moscow, Issue 12, - pp. 52-68 (in Russian).

4. Bolotin, V.V. (1978). Vibration in Equipment, Oscillation of the Linear Systems, - Vol. 1, Publishing Hause // Machinery Construction, Moscow (in Russian).

5. Direction for the Development of Designs of Cutting Apparatus for Agricultural Mechanics (Foreign Practice: Background Information), Bulletin of CSRIITE, Tractor and Agricultural Mechanics, Issue 10, Moscow, 1978 (in Russian).
6. Levendel, E.E. (1981). Vibration in Equipment, Vibration Processes and Machines, - Vol. 4, Publishing Hause//Machinery Construction, Moscow (in Russian).

7. Milne-Thomson, L.M. (1964). // Theoretical Hydrodynamics, Publishing House "Mir", Moscow (in Russian).

8. Prandtl, L. (2000). Hydro- and Aeromechanics (Translated from the Second German Edition of G.A. Volnert), Scientific and Publishing Center // Regular and Chaotic Dynamics, Moscow (in Russian).

9. Tarverdyan, A.P. (1996). Technical and Technological Bases of Designing Cutting Apparatus for Harvesting Machines and Mowers. PhD Thesis, Yerevan (in Russian).

10. Tarverdyan, A.P. (2014). Application of the Vibration Theory in the Agricultural Mechanics. Publishing Hause "Gitutyun", NAS RA, Yerevan (in Russian).

11. Tarverdyan'), A.P., Altunyan, A.V., Baghdasaryan, A.S., Yeghiazaryan, G.M. (2020). Theoretical Research on Vibratory Cutting of the Plants Stems in the Dense Environment: Vibrationless Cutting // Agriscience and Technology, ANAU, 70/2, - pp. 21-28.

12. Tarverdyan ${ }^{2)}$, A.P., Altunyan, A.V., Yeghiazaryan, G.M. (2020). Theoretical Research on Vibratory Cutting of the Plants Stems in the Dense Environment: Cutting with Vibration, Tbilisi // Annals of Agrarian Science, Volume 18, Number 2, - pp. 233-239.

13. Tarverdyan ${ }^{3)}$, A.P., Altunyan, A.V., Baghdasaryan, A.S., Yeghiazaryan, G.M. (2020). Scientific Experimental Research on Plants Stem Vibro-Cutting in the Dense Environment. Tbilisi // Annals of Agrarian Science, Volume 18, - N 3, - pp. 417-423. 